\title{
CORIOAMNIONITIS: EVALUACIÓN DEL RIESGO NEONATAL
}

\author{
Chorioamnionitis: assessment of the neonatal risk
}

\author{
Joseph Alburqueque Melgarejoํ, Juan Carlos Roque Quezada², Juan Carlos Tasayco Saravia³
}

\begin{abstract}
Estimado señor editor:
Luego de haber leído con interés el artículo "Resultados perinatales de embarazos con corioamnionitis en un Hospital de nivel III de Lima, 2016 a 2018” por la Dra Carmen Carolina De La Cruz-Dávila et al publicado en el número Numero 1, Volumen 9 de su revista; el cual consideramos un estudio de amplia relevancia en el campo de la Gineco-Obstetricia y Neonatología; por su extenso impacto en la salud materna y en la morbimortalidad neonatal en el Perú; quisiéramos acotar la importancia de reportar también la retinopatía de la prematuridad (RP) como posible complicación de corioamnionitis en futuros estudios y del análisis anatomopatológico de la placenta como fuerte indicador pronóstico de los recién nacidos con esta patología en futuros estudios de la institución.
\end{abstract}

La RP es un trastorno vasoproliferativo de la retina que ocurre como resultado de una alteración en el desarrollo neurovascular de la retina en el neonato prematuro, que, a través de mecanismos de compensación patológicos conllevan a la proliferación vascular aberrante de la retina. Históricamente esta patología se ha visto asociada en neonatos prematuros que recibían oxígeno a altas concentraciones en la incubadora. Los principales factores de riesgo para el desarrollo de esta entidad son exposiciones a concentraciones altas de oxígeno, prematuridad en el neonato, bajo peso al nacer, hiperglicemia fetal e infecciones neonatales, como la corioamnionitis, además suele presentarse con otras comorbilidades, como alteraciones neurológicas, enterocolitis necrotizante, displasia broncopulmonar y hemorragia intraventricular ${ }^{1,2}$.

En los últimos años, varios estudios de alta confiabilidad mencionan a la corioamnionitis como un posible factor de riesgo para el desarrollo de RP. Debemos ser conscientes, de que esta patología representa una causa potencial de ceguera prevenible en la infancia ${ }^{2,3}$. Se requieren más estudios de tipo prospectivo para determinar la prevalencia de la retinopatía de la prematuridad en neonatos nacidos de madres con corioamnionitis en el Perú.

Por otra parte, en el estudio no se tomaron en cuenta los resultados histopatológicos, esto es de gran relevancia, pues la presencia de corioamnionitis histológica puede influir en el desarrollo fetal. La evidencia histológica de funisitis también podría influir en el resultado de las complicaciones fetales como prematuridad ${ }^{4}$.

La evaluación de la placenta por anatomía patológica, es de vital importancia, pues en el Consenso del Grupo de Trabajo Placentario de Amsterdam del 2014, refieren que la descripción de la topografía de la inflamación (ubicación de la inflamación en las membranas, cordón umbilical o cara fetal de la placenta) permitirá identificar y separar si la respuesta inflamatoria es materna o fetal. Se cree que esto es importante porque hay evidencia que complicaciones fetales severas al nacimiento se asocian más a menudo con una respuesta inflamatoria fetal (compromiso del cordón umbilical). Además la cronicidad de la inflamación puede tener implicaciones clínicas diferentes de las de una respuesta puramente aguda, y por lo tanto debe documentarse ${ }^{5}$.

La presencia de neutrófilos en el espacio intervellosco subcorial, debajo de la capa del corion leve, en ausencia de inflamación en otro lugar, no es sinónimo de corioamnionitis aguda y se debe informar como subcorionitis. Se recomienda, que además de la ubicación topográfica, la inflamación debe clasificarse y estadificarse. La Clasificación propuesta por la Sociedad de Patología Pediátrica proporciona una escala y definiciones para ambos (Coriamnionitis y funisitis) ${ }^{5}$.

Además es importante precisar en casos de respuesta inflamatoria fetal si es que la inflamación compromete la vena o la arteria umbilical. La evidencia sugiere una diferencia en los niveles de citoquinas entre la arteritis umbilical y la flebitis umbilical, y una correlación entre los niveles de citoquinas y el número de vasos implicados. Se han informado tasas más altas de resultados neonatales adversos en neonatos con arteritis umbilical en comparación con los que no tienen ${ }^{5}$.

\footnotetext{
Investigador junior del Instituto de Investigación en Ciencias Biomédicas de la Universidad Ricardo Palma.

Docente de la Universidad Científica del Sur.

3 Jefe del Departamento de Patología del Instituto Nacional Materno Perinatal.
}

Citar como: Alburqueque J, Roque JC, Tasayco JC. Corioamnionitis: evaluacion del riesgo neonatal. Rev Peru Investig Matern Perinat 2020; 9(2): 9-10. DOI https://doi.org/10.33421/inmp.2020197

Recibido: 26-06-20 Aprobado: 30-06-20 
Teniendo en cuenta que el Streptococcus agalactiae o beta hemolítico del grupo B (GBS) según la clasificación de Lancefield representa un patógeno importante en la patogénesis de la corioamnionitis, y teniendo en cuenta estudios como el de Soto et al., que reporta un aumento en el aislamiento de GBS en cultivos de orina, se debería priorizar/optimizar la detección de este patógeno. Hubiera resultado de gran utilidad agregar esta variable en el estudio, pues habría brindado un porcentaje aproximado del número de casos de corioamnionitis atribuida a este agente y podría haber evidenciado si este incremento se correlaciona con la incidencia de corioamnionitis ${ }^{6}$. Se requieren más estudios multicéntricos para evaluar la presencia de GBS en gestantes con corioamnionitis.

Con todo lo expuesto, esta carta pretende incentivar la investigación en las características destacadas previamente, pues a nivel nacional no se cuentan con muchos estudios que hablen del tema. Además, dichas investigaciones podrían servir para tomar nuevas medidas que ayuden a disminuir la incidencia en corioamnionitis en nuestro medio.

Financiamiento: autofinanciado.

Conflicto de intereses: Los autores declaran no tener ningún conflicto de interés.

\section{REFERENCIAS BIBLIOGRÁFICAS}

1. Hellström A, Smith LEH, Dammann O. Retinopathy of prematurity. Lancet. 2013;382(9902):1445-1457. DOI:https:// doi.org/10.1016/S0140-6736(13)60178-6.

2. Souvik M, Dagfinn A. Chorioamnionitis as a Risk Factor for Retinopathy of Prematurity: A Systematic Review and Meta-Analysis. 2014. Neonatology Volume: 105. DOI: $10.1159 / 000357556$

3. Villamor-Martinez E, et al. Chorioamnionitis as a risk factor for retinopathy of prematurity: An updated systematic review and meta-analysis. https://doi.org/10.1371/journal.pone.0205838

4. Palmsten $\mathrm{K}$, et al. Subclinical and Clinical Chorioamnionitis, Fetal Vasculitis, and Risk for Preterm Birth: A Cohort Study. Placenta. 2018;67:54-60. doi: 10.1016/j. placenta.2018.06.001

5. Pulido A, Soto J. Incremento de aislamientos de Streptococcus agalactiae en cultivos de orina en un hospital materno-infantil de Lima, Perú. An Fac Med. [Internet]. 2019 Abr [citado 2020 Jun 22] ; 80( 2 ): 266-267. Disponible en: http:// www.scielo.org.pe/scielo.php?script=sci_arttext\&pid=S1025$55832019000200023 \&$ Ing=es.

6. Khong TY, Mooney EE, et al. Amsterdam Placental Workshop Group Consensus Statement. Sampling and Definitions of Placental Lesions. Arch Pathol Lab Med-Vol 140, July 2016

\section{Correspondencia:}

Juan Carlos Roque Quezada

Direccción: Av. Sucre 623 Dpto. 703 Pueblo Libre

Correo: juankmed26@gmail.com

Teléfono: 945558094 